\title{
Domain Shape Relaxation and the Spectrum of Thermal Fluctuations in Langmuir Monolayers
}

\author{
Raymond E. Goldstein* and David P. Jackson \\ Department of Physics, Joseph Henry Laboratories, Princeton University, Princeton, New Jersey 08544 \\ Received: May 10, 1994; In Final Form: July 8, $1994^{\otimes}$
}

Isolated domains in Langmuir monolayers exhibit shape instabilities leading to branched structures as a consequence of the competing effects of line tension and dipolar interactions. A theory for interfacial pattern formation in the presence of these forces, developed to study shape instabilities of magnetic fluids, is reformulated to treat electric dipolar systems and specialized to the case of "ultrathin" domains, for which the aspect ratio $2 R / h \gg 1$, where $R$ is the domain radius and $h$ its thickness or a microscopic cutoff. Two phenomena studied in recent experiments are considered on the basis of this model: the spectrum of thermal fluctuations of the domain boundary and the dynamics of shape relaxation near the branching instability. The experimental spectrum of thermal fluctuations for monolayers of dimyristoylphosphatidylcholine and cholesterol (Seul, M. Physica A 1990, 168, 198) deviates in a small but measurable way from that expected in the presence of line tension alone and is described quantitatively by the theory. This analysis yields estimates for the line tension and dipole moment density which are in accord with previous determinations by other methods. The relaxation of branched shapes to a circular ground state is found, as in experiment (Seul, M. J. Phys. Chem. 1993, 97, 2941), to deviate in a characteristic way from the "curve-shortening" law which governs the motion in the absence of dipolar interactions. A heuristic argument explaining this phenomenon, in which the notion of a scale-dependent surface tension is introduced, is formulated on the basis of a "localized induction approximation". This approximation, familiar from the study of vortex motion in inviscid hydrodynamics, is applied here to the Biot-Savart integrals which represent the dipolar pressure at the boundary of the domain. A relationship between the energetics of fingering instabilities and domain fission is proposed on the basis of analytical results obtained in the ultrathin limit. Details of a numerical method for the study of this shape evolution are provided, with particular attention paid to a consistent treatment of cutoff effects.

\section{Introduction}

Recent experimental studies ${ }^{1-6}$ of pattern formation in Langmuir monolayers have focused on dynamical aspects of domain shape evolution. These include investigations of the thermal fluctuations of domain boundaries, analogous to capillary waves at a fluid-air interface, as well as the relaxation of large-amplitude shape distortions brought on by fingering instabilities. These instabilities, in turn, have been suggested to arise from the competition between line tension and longrange dipolar interactions between the amphiphiles. ${ }^{7-14}$

It has been suggested ${ }^{12,14}$ that the energetic competition between surface tension and dipolar forces in amphiphilic monolayers should be essentially equivalent to that of domains of magnetizable fluids ${ }^{15}$ trapped between closely spaced plates (the geometry of Hele-Shaw flow). Experiments on the latter ${ }^{15-18}$ have revealed that when a magnetic field is applied normal to the plates, orienting the microscopic magnetic moments in suspension, the domain undergoes fingering instabilities exactly like those of the amphiphilic domains.

Here we apply a recent approach ${ }^{14}$ to the energetics and dynamics of pattern formation in magnetic fluids to compute the spectrum of thermal fluctuations for circular domains and the dynamics of shape relaxation in Langmuir monolayers. An important distinction between the magnetic and amphiphilic systems concerns the relationship of two characteristic lengths in the system: the radius $R$ of the circular domain and its thickness $h$ (the spacing between plates in the Hele-Shaw problem or a microscopic cutoff in the case of the monolayers).

\footnotetext{
${ }^{\otimes}$ Abstract published in Advance ACS Abstracts, August 15, 1994.
}

It is convenient to define an aspect ratio

$$
p=2 R / h
$$

the range of which is $10 \leq p \leq 100$ for ferrofluid domains. For the typical phospholipid domain whose radius is $50 \mu \mathrm{m}$ and with a molecular cutoff of $h \simeq 1-10 \AA$ the relevant range of aspect ratios is $10^{5} \leq p \leq 10^{6}$. We refer to this as the "ultrathin limit".

Specializing to the ultrathin limit, we first derive in section II the limiting form of the dipolar energy of a domain of arbitrary shape and use equipartition considerations to obtain a closedform expression for the amplitudes of thermally excited modes of circular domains and the related linear stability analysis. The range of validity of the results for $p \rightarrow \infty$ is discussed, and some further analytical approximations useful in that limit, discussed briefly in the context of the hydrodynamics of Hele-Shaw flow of dipolar fluids, ${ }^{19}$ are presented. The fluctuation predictions are compared with recent experimental results ${ }^{4}$ in section III, where we obtain estimates of the line tension $\gamma$ and discontinuity in dipole moment per unit area $\mu$ between the coexisting phases for a particular monolayer composition. These estimates complement earlier measurements of these parameters by different techniques. ${ }^{1,2}$ We may then compute the dimensionless Bond number,

$$
N_{\mathrm{B}}=2 \mu^{2} / \gamma
$$

the energetic control parameter characterizing the relative importance of dipolar and surface energies. The available data is not sufficient to provide a stringent test of the theory, but 
make it possible to locate approximately the experimental system in a stability diagram in the $p-N_{\mathrm{B}}$ plane.

Quite naturally, the problem of small-amplitude fluctuations about a simple shape is closely connected with the linear stability analysis, and the theoretical results discussed here should be viewed as complementary to several recent stability analyses ${ }^{13,20}$ which differ in subtleties regarding the implementation of the cutoff.

The results on the thermal fluctuations provide a test of the proposed energy functional for dipolar domains, but do not address directly the dynamics of their boundaries. For this, we turn in section IV to a study of the relaxation toward a circular shape of a branched domain. This is done within the context of the simplest dissipative dynamics, which is a gradient flow in the space of configurations. ${ }^{14}$ Measurements of shape relaxation ${ }^{5}$ have shown deviations from the behavior predicted in the presence of line tension alone and were attributed to dipolar forces. We show that the form of these deviations is consistent with the dissipative dynamics of dipolar systems when the domain is near the branching instability. In turn, these data provide justification for the heuristic notion of a scale-dependent effective surface tension in dipolar systems. This concept is developed further here using the localized induction approximation on the Biot-Savart integrals representing the dipolar pressure at the domain boundary. This approximation is wellknown in the study of vortex motion in fluid mechanics. ${ }^{21.22}$ Along with recent experimental studies of shape instabilities ${ }^{23,24}$ these results begin to allow for a precise comparison between theory and experiment. Suggestions are made for a particular series of experiments to test quantitatively the predictions of these models. In section $\mathrm{V}$ we comment on the relationship between the balance of forces embodied in the linear stability analysis and simple energetic considerations involving the fissioning of domains, establishing a simple intuitive picture of the origin of branching instabilities.

Some further avenues of investigation are suggested in the concluding section VI. Appendices A and B collect a number of details regarding the computation of thermal fluctuations and of the numerical method used to study the shape evolution, with particular attention to a consistent treatment of the cutoff contributions to the energy and dynamics.

\section{Energetics and Thermal Fluctuations in the Ultrathin Limit}

Consider a thin dipolar domain of height $h$, whose base lies in the $x-y$ plane and whose boundary is described by the contour $\mathcal{G}$. The total energy $\mathcal{B}$ is the sum of contributions from line tension and electrostatic interactions,

$$
\mathcal{E}=\mathcal{E}_{\gamma}+\mathcal{E}_{\mu}
$$

If the length of $O$ is $L$ and the surface tension between the phases interior and exterior to the domain is $\sigma$, then clearly $\mathscr{E}_{\gamma}=\gamma L$, where the line tension $\gamma=\sigma h$. In the simplest model for the dipolar energy of such a domain, the dipole moment per unit area is taken to be uniform both within and external to the domain. Let $\mu$ be the discontinuity of the dipole density across the boundary $\mathcal{G}$. As shown elsewhere, ${ }^{12,14} \mathcal{E}_{\mu}$ is the sum of a term proportional to the area $A$ of the domain and a contribution in the form of the self-induction of the boundary,

$$
\mathscr{E}_{\mu}[\mathbf{r}]=\frac{2 \pi \mu^{2}}{h} A-\frac{\mu^{2}}{h} \oint_{\mathrm{d} s} \oint_{\mathrm{d} s^{\prime}} \hat{\mathbf{t}}(s) \cdot \hat{\mathbf{t}}\left(s^{\prime}\right) \Phi(R / h)
$$

Here, $\mathbf{r}(s)$ is the location of a point on $G$ at arc length $s, \hat{\mathbf{t}} \equiv$ $\partial \mathbf{r} / \partial s$ is the unit tangent vector there, and $R=\left|\mathbf{r}(s)-\mathbf{r}\left(s^{\prime}\right)\right|$.
The function

$$
\Phi(\xi)=\ln \left(\frac{1}{\xi}+\left(1+\frac{1}{\xi^{2}}\right)^{1 / 2}\right)+\xi-\left(1+\xi^{2}\right)^{1 / 2}
$$

arises from integrations over the thickness of the domain and may be thought of as producing a "smooth" cutoff to the otherwise divergent behavior of the energy for $s$ near $s^{\prime}$ in eq 2.2. Of interest in later sections is the exact dipolar energy for circular domains ${ }^{14,25}$

$$
\begin{array}{r}
\mathscr{E}=\frac{2 \pi \mu^{2}}{h} A+\frac{\mu^{2}}{3 \pi h^{2}} R^{3}\left\{1-k^{-3}\left[\left(2 k^{2}-1\right) E(k)+\right.\right. \\
\left.\left.\left(1-k^{2}\right) K(k)\right]\right\}
\end{array}
$$

with $K$ and $E$ being complete elliptic integrals and $k^{2}=p^{2} /(1$ $+p^{2}$ ). For large $p$ we obtain the limiting behavior

$$
\mathcal{G}_{\mu} \simeq \frac{2 \pi \mu^{2}}{h} A-2 \pi \mu^{2} R \ln \left(\frac{8 R}{e^{1 / 2} h}\right)
$$

We may now turn to the computation of the energy of a circular domain perturbed by small amplitude radial distortions and determine their mean amplitudes. With $\varphi$ being the polar angle and $\hat{\mathbf{e}}_{r}(\varphi)$ a unit vector in the radial direction, we describe the radius of the domain as

$$
\mathbf{r}(\varphi)=(R+\zeta(\varphi)) \hat{\mathbf{e}}_{r}(\varphi)
$$

Writing $\zeta(\varphi)=\Sigma_{n} \xi_{n} \cos (n \varphi)$, the energy may be written up to quadratic order as

$$
\mathscr{E}[\mathbf{r}]=\mathscr{E}_{\text {circle }}+\frac{1}{2} \sum_{n=1}^{\infty} \Omega_{n} \zeta_{n}^{2}
$$

and our goal is to compute $\Omega_{n}$. Experiments on thermal fluctuations reveal that the area of the domain is essentially constant over the duration of the experiment. ${ }^{5}$ The details of the calculation of $\Omega_{n}$ under this constraint are given in Appendix A. We obtain

$$
\Omega_{n} \simeq(\pi \gamma / R) \beta_{n}
$$

where

$$
\begin{aligned}
\beta_{n}\left(N_{\mathrm{B}}, R, h\right)=\left[1-\frac{1}{2} N_{\mathrm{B}} \ln \left(\frac{8 R}{e h}\right)\right] & \left(n^{2}-1\right)+ \\
& \frac{1}{4} N_{\mathrm{B}}\left(1-4 n^{2}\right) \sum_{j=2}^{n} \frac{1}{2 j-1}
\end{aligned}
$$

a central result for what follows.

As long as all amplitudes $\Omega_{n}$ are positive, the circle is locally stable to small perturbations. With increasing $\mu^{2}$ some $\Omega_{n}$ will become negative; the first to do so will be $\Omega_{2}$ at the critical value

$$
N_{\mathrm{B}} *(2)=\frac{2}{\ln (8 R / h)-11 / 6}
$$

A comparison between the predictions of eq 2.10 and the exact expression $^{26}$ for the instability of the $n=2$ mode, shown in Figure 1, reveals that it is highly accurate even for $p \sim 10$. The inset to Figure 1 shows $\Omega_{n}$ for $p=10^{5}$ and various values of $N_{\mathrm{B}}$ near $N_{\mathrm{B}}{ }^{*}(2)=0.1807$, illustrating the rapid appearance of a broad band of unstable modes with increasing dipolar strength. 


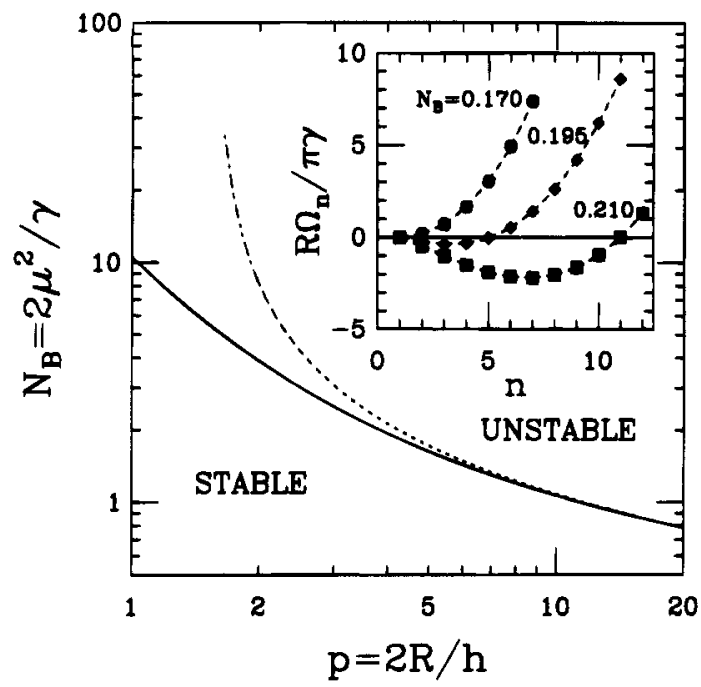

Figure 1. Stability diagram for dipolar domains in the ultrathin limit, Solid line is exact stability boundary for the $n=2$ mode, ${ }^{26}$ while dashed line is the large- $p$ approximation in eq 2.10. Inset shows mode amplitudes $\Omega_{n}$ for various dipolar Bond numbers, for $p=10^{5}$, in the ultrathin limit of eq 2.9 .

In the limit of large aspect ratios the cutoff $h$ enters into the shape instabilities and dynamics only in a rather indirect way, for instance determining an effective line tension as in eq 2.9. It is only when the length scale of the pattern approaches the cutoff that significant effects occur. In particular, the most unstable wavelength of a circular domain and the equilibrium width of a stripe both acquire a nontrivial dependence on the cutoff. Such behavior is unlikely to be observed in Langmuir monolayers, where the cutoff is molecular. This regime can be accessed in the study of macroscopic domains of magnetic fluids, as will be reported elsewhere. ${ }^{27}$

Equation 2.9 may be used to define the exact limiting form (as $p \rightarrow \infty$ ) of the critical Bond number $N_{\mathrm{B}}{ }^{*}(n)$ at which the $n$th mode becomes energetically favored. This is not, in general, the Bond number at which the mode $n$ is the most unstable (except for the case $n=2$ in eq 2.10), but it is closely related. In the ultrathin limit it has the simple form

$$
N_{\mathrm{B}} *(n)=\frac{2}{\ln (8 R / h)-g(n)}
$$

where

$$
g(n)=1+\frac{1}{2}\left(\frac{4 n^{2}-1}{n^{2}-1}\right) \sum_{j=2}^{n} \frac{1}{2 j-1}
$$

These results for the critical Bond number may be reexpressed in terms of the critical radius $R^{(n)}$ for the instability of the $n$th mode, at a given value of $N_{\mathrm{B}}$. From eq 2.11 one finds

$$
R^{(n)}=(h / 8) \mathrm{e}^{\gamma / \mu^{2}} \mathrm{e}^{g(n)}
$$

The function $g(n)$ is shown in Figure 2. One may verify in detail that these limiting forms are completely consistent with the known exact results for the first few modes, for arbitrary $p .26$

The form of the relation 2.13 is identical to that derived by McConnell ${ }^{28}$ within an approximation involving a sharp cutoff. It implies the very simple relation for the first two modes

$$
R^{(3)} / R^{(2)}=\mathrm{e}^{1 / 3} \simeq 1.396
$$

a result arrived at independently by Cebers, ${ }^{29}$ in agreement with

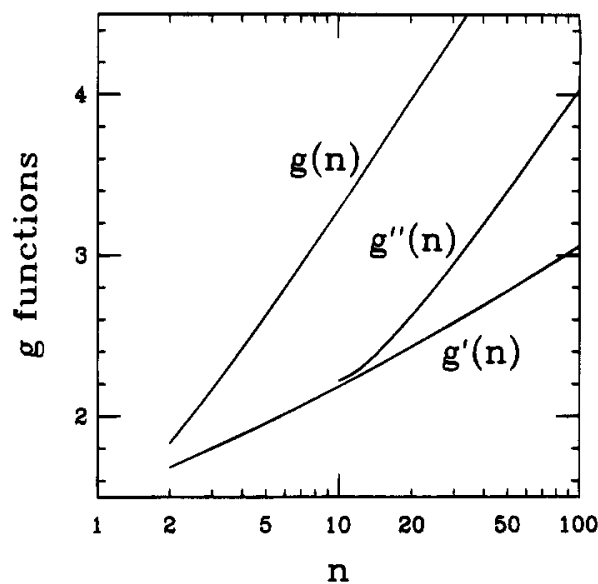

Figure 2. The quantity $g(n)$ entering the linear stability analysis (eq 2.12) and approximations $g^{\prime}(n)$ and $g^{\prime \prime}(n)$ (egs 5.5 and 5.9) derived from the energetics of fission.

the results of Lee and McConnell obtained using a sharp-cutoff approximation, and consistent with experiment. ${ }^{23}$

Provided $N_{\mathrm{B}}<N_{\mathrm{B}}^{*}(2)$, so all $\beta_{n}>0$, we may appeal to equipartition to obtain the thermal average of the squared amplitude as

$$
\left\langle\zeta_{n}^{2}\right\rangle=\frac{k_{B} T R}{\pi \gamma \beta_{n}\left(N_{\mathrm{B}}, R, h\right)}
$$

If $\mu=0$ we obtain $\left\langle\zeta_{n}{ }^{2}\right\rangle \propto 1 /\left(n^{2}-1\right)$, a result noted by Seul and Sammon. ${ }^{3}$ It should be emphasized that the result 2.15 , based on eq 2.7 , will eventually break down close enough to the branching instability, when $\Omega_{n} \rightarrow 0$, the mode amplitudes grow large, and thus terms beyond quadratic order in the energy functional become important. A detailed computation of those has not yet been undertaken, but would be of interest in determining whether the branching instability is a subcritical or supercritical transition. We now turn to a direct comparison of eqs 2.9 and 2.15 with experimental measurements.

\section{Thermal Fluctuations: Comparison with Experiment}

In comparing with experiment, we use the data of Seul, ${ }^{4}$ obtained for dimyristoylphosphatidylcholine (DMPC) monolayers with $30 \%$ cholesterol and $1 \%$ fluorescent dye. The three data sets of the equilibrium mean-square mode amplitudes $\left\langle\zeta_{n}{ }^{2}\right\rangle$ for three domain radii $(R=67.3,61.7$, and $62.2 \mu \mathrm{m})$ are shown in Figure 3. We see that the typical amplitudes of fluctuations in the low-order modes are on the order of 1-2 $\mu \mathrm{m}$ and fall off rapidly with order.

We now wish to extract from these data estimates for the Bond number $N_{\mathrm{B}}=2 \mu^{2} / \gamma$. As is apparent from the form of eq 2.9 for the quantities $\Omega_{n}$, we must specify the microscopic length scale $h$. Since we have no a priori knowledge of an appropriate value, we have explored a range of molecular scales $1 \AA \leq h$ $\leq 10 \AA$, finding the value of $N_{\mathrm{B}}$ for each that gives a good fit to the data. It transpires that the values obtained for $\gamma$ and $\mu$ do not depend sensitively on the assumed value of $h$.

A convenient replotting of the data is shown in Figure 4 for the three data sets: the fluctuation amplitudes $C_{n} \equiv\left\langle\xi_{n}{ }^{2}\right\rangle$ normalized by $C_{2}$, plotted versus $n^{2}-1$. We see from eqs 2.8 and 2.9 that $C_{2} / C_{n}=\beta_{n} / \beta_{2}$ depends only on the Bond number and aspect ratio, and not separately on $\mu$ or $\gamma$. Were there only line tension acting on the boundary, then such a plot would be exactly linear, with slope $1 / 3$ and zero intercept, as indicated by the dashed line in the figure. Instead we see a small amount 


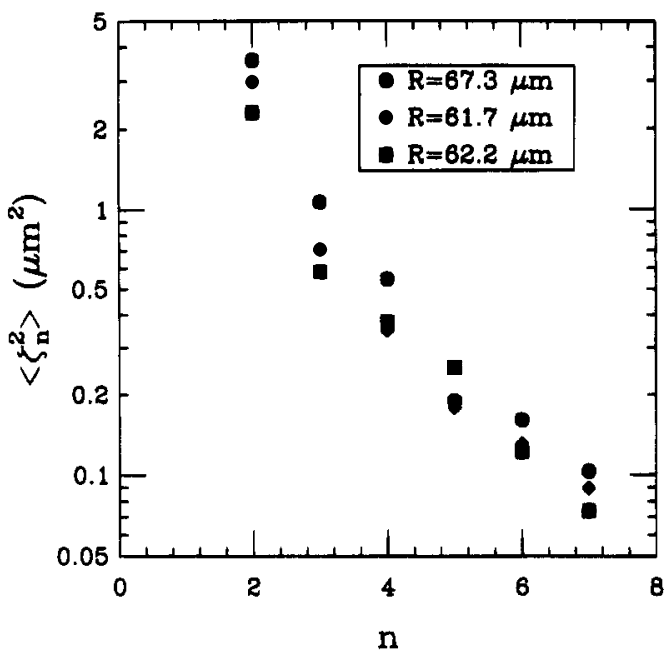

Figure 3. Amplitudes of thermal fluctuations of domains of three radii in a monolayer of DMPC and cholesterol (30\%) (from ref 4).

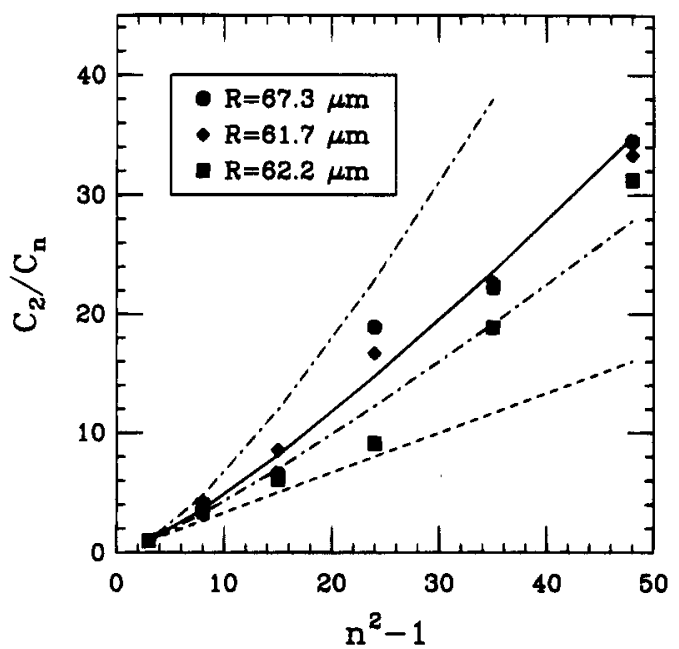

Figure 4. Data of Figure 3 replotted in dimensionless form. Deviation from dashed-straight-line behavior (lowest dashed curve) indicates the presence of dipolar forces. Solid line is a fit to eq 2.15 with $h=1 \AA$, and $N_{\mathrm{B}}=0.137$, appropriate to the domain with $R=67.3 \mu \mathrm{m}$. Dotdashed lines indicate sensitivity of the theoretical curve to changes in $N_{\mathrm{B}}$ of \pm 0.005 from the fitted value.

TABLE 1: Estimates of the Dipolar Bond Number $N_{B}$ for Different Values of the Cutoff

\begin{tabular}{cccc}
\hline$R(\mu \mathrm{m})$ & $h=1 \AA$ & $h=5 \AA$ & $h=10 \AA$ \\
\hline 67.3 & 0.137 & 0.154 & 0.163 \\
61.7 & 0.137 & 0.154 & 0.163 \\
62.2 & 0.134 & 0.150 & 0.158
\end{tabular}

of curvature, a manifestation of the competition between terms scaling as $n^{2}$ and as $n^{2} \ln (n)$. All three data sets cluster close together when plotted as in Figure 4.

For each of the three domain radii, and each of three representative values of the cutoff $h(1,5$, and $10 \AA)$, we have obtained by nonlinear least squares fitting the best-fit value of the Bond number, as summarized in Table 1. The solid line in Figure 4 illustrates the quality of such fits and the sensitivity of the fit to very small changes in the Bond number.

With a fitted value of $N_{\mathrm{B}}$ for each choice of cutoff we may estimate the bare line tension by the relation

$$
\gamma=\frac{R k_{B} T}{\pi \beta_{n} C_{n}}
$$

Figure 5 serves the dual purpose of illustrating the residuals of

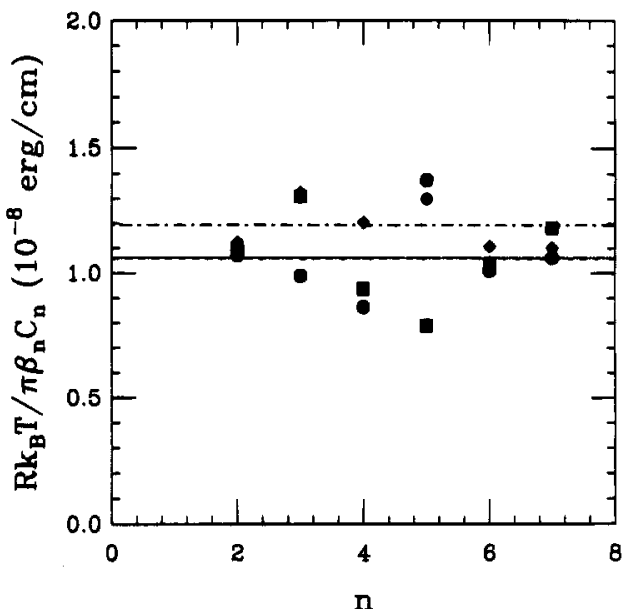

Figure 5. Estimates of the line tension for the three data sets of Figure 3 , following eq 3.1 with $h=10 \AA$. Horizontal lines are at mean values as quoted in Table 2.

TABLE 2: Estimates of the Bare Line Tension $\gamma$ for Different Values of the Cutoff, in Units of $10^{-8} \mathrm{erg} / \mathrm{cm}$

\begin{tabular}{cccc}
\hline$R(\mu \mathrm{m})$ & $h=1 \AA$ & $h=5 \AA$ & $h=10 \AA$ \\
\hline 67.3 & 1.26 & 1.12 & 1.06 \\
61.7 & 1.42 & 1.26 & 1.19 \\
62.2 & 1.25 & 1.12 & 1.06
\end{tabular}

the fit in Figure 4 and those for the other two domains, as well as showing the mean values of $\gamma$ around which each of those data sets cluster. Table 2 summarizes those fits.

Finally, given $N_{\mathrm{B}}$ and $\gamma$ we obtain estimates for the dipole moment density $\mu$. Choosing $h=10 \AA$ as a representative value we conclude that

$$
\gamma \simeq(1.1 \pm 0.1) \times 10^{-8} \mathrm{erg} / \mathrm{cm}
$$

and

$$
\mu \simeq 0.30 \pm 0.02 \mathrm{D} / 100 \AA^{2}
$$

The value for the line tension is roughly consistent with that obtained by Benvegnu and $\mathrm{McConnel}^{2}$ for pressures near the critical point of the DMPC-cholesterol mixture. The dipole density is considerably lower than their estimate $\mu \simeq 0.7 \mathrm{D} / 100$ $\AA^{2}$, obtained at very low surface pressure. The present data were obtained much closer to the critical point, so the density difference between the coexisting phases would be much smaller. A quantitative verification of this point awaits further experimental study.

It is of interest to note from eq 2.9 that one may define an effective line tension $\tilde{\gamma}$ in the presence of dipolar forces,

$$
\tilde{\gamma}=\gamma\left[1-\frac{1}{2} N_{\mathrm{B}} \ln \left(\frac{8 R}{e h}\right)\right]
$$

whose vanishing defines approximately the onset of the branching instability. Using the parameters extracted with $h=10 \AA$, we find the extremely low value

$$
\tilde{\gamma} \simeq(0.01-0.04) \times 10^{-8} \mathrm{erg} / \mathrm{cm}
$$

This proximity of the experimental systems to the boundary of shape instabilities is illustrated in Figure 6, which plots the fitted Bond numbers obtained over a very broad range of aspect ratios (reflecting different choices of $h$ ) for the data on domain 2 . We see that a variation of 2 orders of magnitude in $p$ is associated only with a $50 \%$ change in the estimate of $N_{\mathrm{B}}$. Moreover, for all values of $h$ the data are seen to lie quite close to the stability 


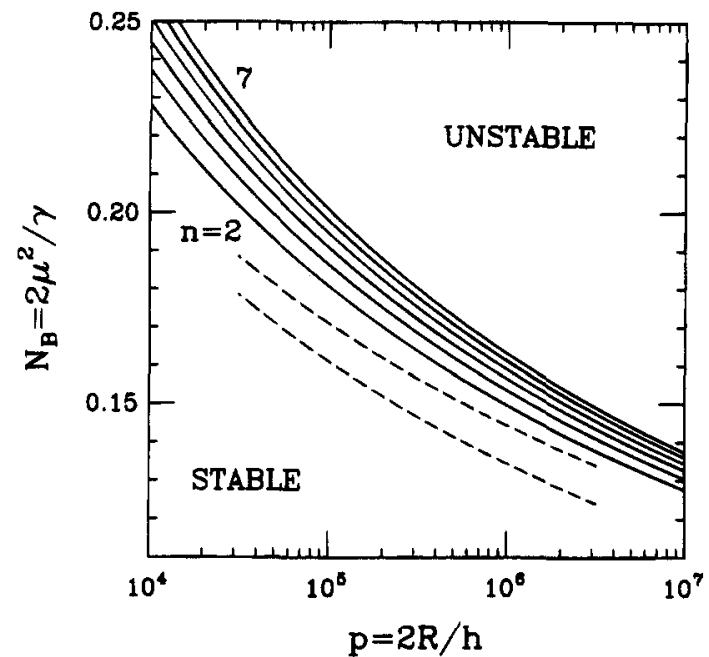

Figure 6. Locating experiments on the stability diagram. Dashed lines represent a spread of \pm 0.005 of the fitted values of $N_{\mathrm{B}}$ on a domain of radius $61.7 \mu \mathrm{m}$, using values of the cutoff $h$ ranging over several decades. Solid lines indicate the locus of instabilities for modes $n=$ $2-7$.

boundary, a proximity in qualitative accord with the experimental observations.

Clearly, a more stringent experimental test of the validity of the predictions 2.8 and 2.9 for the spectrum of thermal fluctuations requires data covering a range of conditions closely approaching the branching instability. The small curvature seen in Figure 4 is predicted to become more and more pronounced as $N_{\mathrm{B}} \rightarrow N_{\mathrm{B}}{ }^{*}(2)$. This suggests a natural set of experiments in which the spectrum of fluctuations is monitored as the shape transition is approached, by increasing either the temperature or the applied pressure. ${ }^{2}$

\section{Shape Relaxation in the Ultrathin Limit}

In this section we turn to the dynamics of the relaxation of branched domains to the circular ground state, with particular interest in the role of the long-range dipolar forces. Perhaps the simplest model for the dynamics of shape relaxation is one which ascribes all dissipation to the boundary of the domain and balances the viscous force there to the generalized pressure. ${ }^{14}$ When expressed in terms of the normal component of the velocity $\partial \mathbf{r} / \partial t$ of the boundary $\mathbf{r}(s)$, this "dissipative dynamics" equation of motion takes the form

$$
\hat{\mathbf{n}} \cdot \frac{\partial \mathbf{r}}{\partial t}=-\eta \hat{\mathbf{n}} \cdot \frac{\delta \mathcal{E}}{\delta \mathbf{r}}
$$

where $\eta$ is a friction coefficient. The constraint of area conservation is achieved by introducing a Lagrange multiplier $\Pi$ in a Legendre transformed energy

$$
\mathcal{B}=\mathcal{G}_{\mu}+\mathcal{B}_{\gamma}-\Pi A
$$

The variational principle 4.1 then yields an equation of motion of the form

$$
\mathbf{r}_{t}=U \hat{\mathbf{n}}+W \hat{\mathbf{t}}
$$

where

$$
U=\eta\left(U_{\gamma}+U_{\mu}+\Pi\right)
$$

with $U_{\gamma}=-\gamma \kappa, U_{\mu}$ is given in eq $\mathrm{A} 7$, and the tangential velocity $W$ is discussed below. Conservation of area implies that the mean normal velocity vanishes, $\oint \mathrm{d} s U=0$, yielding a nonlocal expression for the Lagrange multiplier,

$$
\Pi=-\left\langle U_{\gamma}\right\rangle-\left\langle U_{\mu}\right\rangle
$$

where the mean of any quantity is defined as $\langle m\rangle \equiv(1 / L) \oint \mathrm{d} s^{m}$. The typical scales of these pressures can be seen from the exact results for circles, for which the simplified relations

$$
\Pi(R)=\frac{1}{2 \pi R} \frac{\partial \mathcal{G}(R)}{\partial R}
$$

hold. This yields

$$
\Pi_{\gamma}(R)=\frac{\gamma}{R}, \quad \Pi_{\mu}(R)=\frac{\mu^{2}}{R} \ln \left(\frac{8 R e^{1 / 2}}{h}\right)
$$

Under the dynamics 4.1 the boundary length $L$ varies with time as ${ }^{30}$

$$
\begin{aligned}
\frac{\partial L}{\partial t} & =\oint_{\mathrm{d} s \kappa}\left(U_{\gamma}+U_{\mu}+\Pi\right) \\
& =\oint_{\mathrm{d} s}\left(\kappa(s)-\frac{2 \pi}{L}\right)\left(U_{\gamma}+U_{\mu}\right)
\end{aligned}
$$

where the second equality follows from the relation $\phi \mathrm{d} s \kappa=$ $2 \pi$ for simple closed curves. Using the explicit forms of $U_{\gamma}$ and $U_{\mu}$, eq 4.8 may be written as

$$
\begin{aligned}
\frac{1}{\eta} \frac{\partial L}{\partial t}= & \gamma\left\{\frac{(2 \pi)^{2}}{L}-\oint_{\left.\mathrm{d} s \kappa^{2}\right\}}+\right. \\
& \frac{2 \mu^{2}}{h^{2}} \oint_{\mathrm{d} s} \oint_{\mathrm{d} s^{\prime}}\left(\kappa(s)-\frac{2 \pi}{L}\right) \frac{\left(\mathbf{r}\left(s^{\prime}\right)-\mathbf{r}(s)\right)}{\left|\mathbf{r}\left(s^{\prime}\right)-\mathbf{r}(s)\right|} \times \\
& \hat{\mathbf{t}}\left(s^{\prime}\right)\left\{\left(1+\frac{h^{2}}{R^{2}}\right)^{1 / 2}-1\right\}
\end{aligned}
$$

The validity of the linear relation between $\partial L / \partial t$ and $(2 \pi)^{2} / L-$ $\oint \mathrm{d} s \kappa^{2}$, which holds for motion by line tension alone, has been tested by Seul ${ }^{4}$ in the relaxation of a branched shape to a circle. Deviations from linear behavior have been observed and were attributed to the presence of dipolar interactions.

To determine if those deviations are consistent with the predictions of eq 4.9 for finite $N_{\mathrm{B}}$, and to study the approach to equilibrium from noncircular shapes, we have performed numerical studies on the relaxation of a branched dipolar domain for values of the Bond number near the critical value $N_{\mathrm{B}}{ }^{*}(2)$. The numerical methods are summarized in Appendix B. For these simulations, we find it convenient to choose units of time and length such that we may set $\gamma=\eta \equiv 1$. The parameters of interest are then $N_{\mathrm{B}}$, the domain area $A$, and cutoff $h$. To characterize the circular state to which a branched structure of area $A$ may flow, we define an "equivalent radius" $R_{0}$ as $R_{0}=$ $(A / \pi)^{1 / 2}$ and specify the cutoff in terms of the equivalent aspect ratio $p=2 R_{0} / h$.

Figure 7a shows the shape relaxation obtained for aspect ratio $p=10^{5}, R_{0}=1.0$, and $N_{\mathrm{B}}=0.175$, just slightly below the critical value $N_{\mathrm{B}}{ }^{*}(2)=0.1807$. The images displayed are at times $t=0,3,6, \ldots$ The dynamics generating this evolution is by construction a gradient flow, and so we may follow the downward flow of energy in time. Shown below in Figure $7 \mathrm{~b}$ and its inset are the total energy $\mathscr{G}_{y}+\mathscr{B}_{\mu}$ and the dipolar contribution alone. Note from this figure and the scale of the perimeter that both $\mathscr{G}_{\gamma}$ and $\mathscr{S}_{\mu}$ are very large in magnitude in comparison with their sum. 
(a)

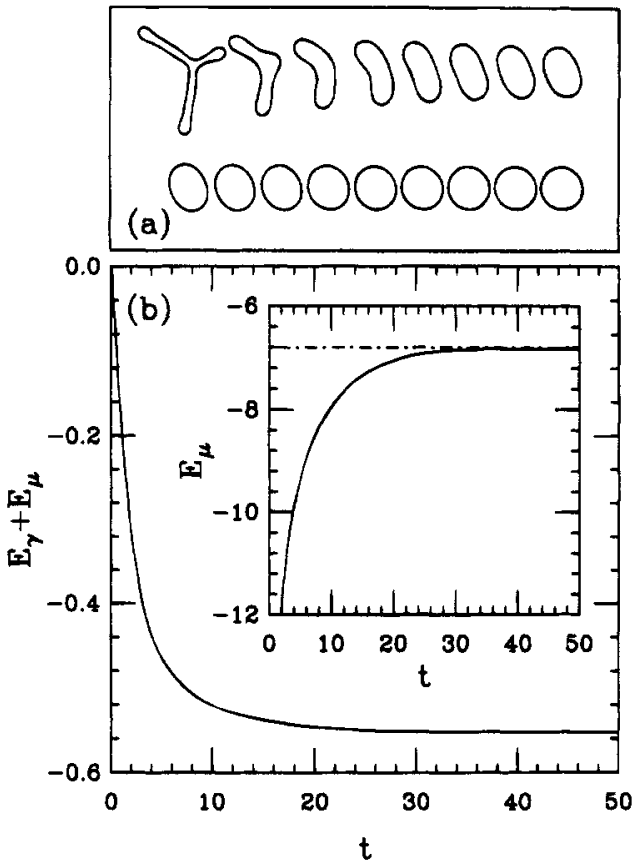

Figure 7. Shape relaxation (a) below the critical Bond number for the 2-fold shape instability. Simulations are for $p=10^{5}, N_{\mathrm{B}}=0.175$, $R_{0}=1.0$, and with $N=256$ Fourier modes; (b) time evolution of total energy and dipolar contribution alone (inset). Dashed line in inset shows value for a circle.

By increasing $N_{\mathrm{B}}$ to 0.182 , now slightly greater than $N_{\mathrm{B}} *(2)$, the same initial condition as in Figure 7a now relaxes (Figure 8 a) to an elongated structure much like that seen in recent experiments. ${ }^{23}$ The associated total and dipolar energies (Figure $8 \mathrm{~b}$ and inset) are each much lower than those of the equivalent circle. The dipolar contribution is actually rather close to that of two isolated domains each with half the area of the original (cf. section $\mathrm{V}$ below).

The plot in Figure 9 shows for the shape relaxation in Figure $7 a$ the rate of change of the perimeter with time as a function of the line tension contributions in eq 4.9. We see clear deviations from the straight-line behavior that would hold in the absence of dipolar interactions, and a form qualitatively like that seen in experiment. ${ }^{5}$ At the early stages of shape evolution there is a reasonably linear relation observed. As the middle branch of the shape disappears, the plot shows marked curvature. One may say that there is a large effective tension in the early stages of the relaxation, when the dominant motion is at the tips of the fingers, and a smaller tension when the interface is less highly curved.

This notion of a scale-dependent line tension is of course at the heart of the linear stability analysis, eqs 2.9 and 3.4. Within the context of the perimeter evolution law (eq 4.9) we may see this explicitly by means of a so-called localized induction approximation (LIA) known from the study of Biot-Savart interactions in the hydrodynamics of vortices and vortex patches in ideal fluids. ${ }^{21,22}$ In this approximation, we first examine the properties of the Biot-Savart integrand in eq 4.9 for $s^{\prime}$ near $s$ and use the Frenet-Serret equations,

$$
\frac{\partial}{\partial s}\left(\begin{array}{l}
\hat{\mathrm{n}} \\
\hat{\mathrm{n}}
\end{array}\right)=\left(\begin{array}{ll}
0 & -\kappa \\
\kappa & 0
\end{array}\right)\left(\begin{array}{l}
\hat{\mathrm{t}} \\
\hat{\mathrm{n}}
\end{array}\right)
$$

to expand the vector $\mathbf{r}\left(s^{\prime}\right)$ as

$$
\mathbf{r}\left(s^{\prime}\right)-\mathbf{r}(s) \simeq\left(s^{\prime}-s\right) \hat{\mathbf{t}}(s)-\frac{1}{2}\left(s^{\prime}-s\right)^{2} \kappa(s) \hat{\mathbf{n}}(s)+\ldots
$$

and conclude that for nearby points on the curve the cross

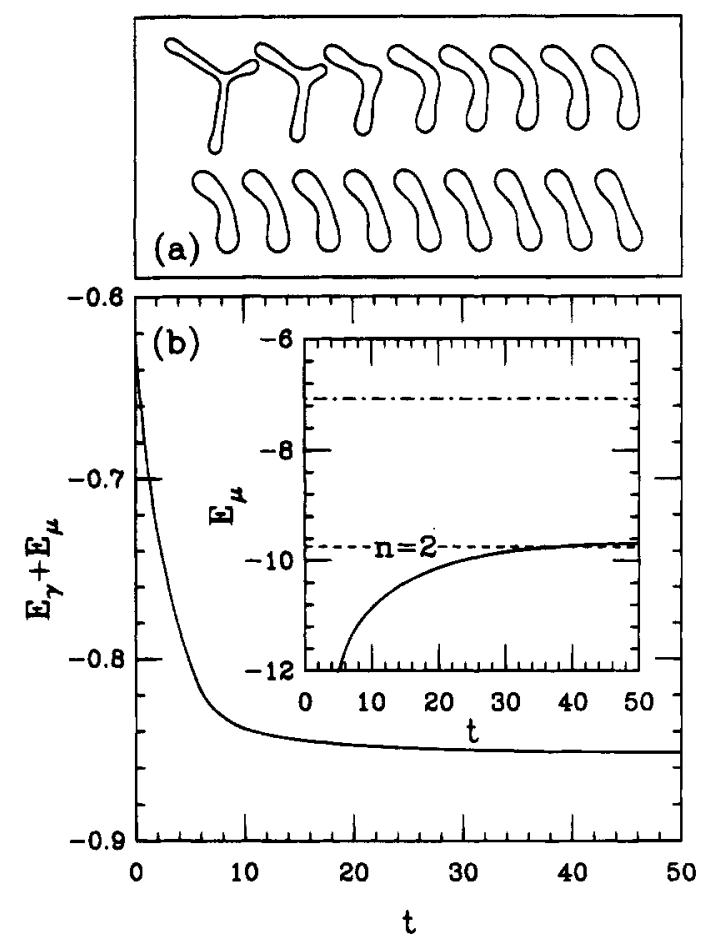

Figure 8. As in Figure 7, but with $N_{\mathrm{B}}=0.182>N_{\mathrm{B}} *(2)$. Additional dashed line in the inset to part $\mathrm{b}$ indicates the dipolar energy of the $n$ $=2$ fissioned configuration.

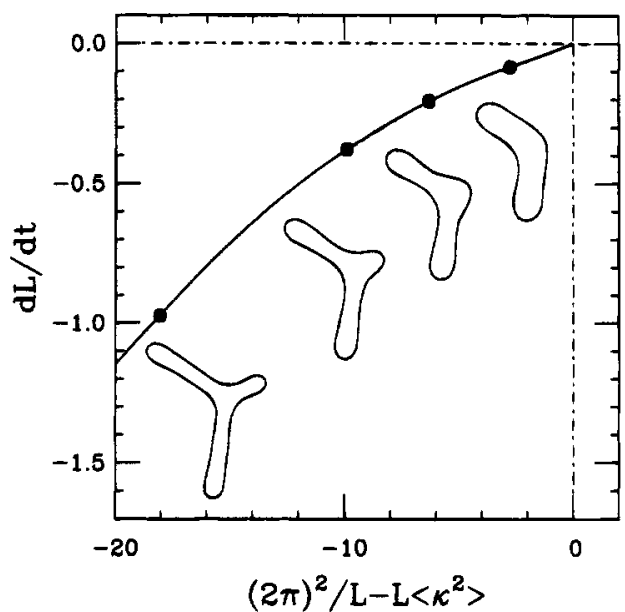

Figure 9. Curve-shortening in the presence of dipolar forces, for the shape evolution in Figure 7. The time derivative of the perimeter is plotted as a function of the first two terms in eq 4.9 to illustrate deviations from the behavior associated with line tension alone. The instantaneous shape is shown at various points in the relaxation.

product has the form

$$
\left(\mathbf{r}\left(s^{\prime}\right)-\mathbf{r}(s)\right) \times \hat{\mathbf{t}}\left(s^{\prime}\right) \simeq \frac{1}{2}\left(s^{\prime}-s\right)^{2} \kappa(s)+\ldots
$$

The second step in the LIA is to introduce a cutoff $\Lambda(s) \gg$ $h$ to the $s^{\prime}$ integral in eq 4.9 and perform the integral explicitly within the approximation 4.12. As discussed in Appendix B, the result is

$$
\oint_{s-\Lambda}^{s+\Lambda} \mathrm{d} s^{\prime} \frac{\left(\mathbf{r}\left(s^{\prime}\right)-\mathbf{r}(s)\right)}{\left|\mathbf{r}\left(s^{\prime}\right)-\mathbf{r}(s)\right|} \times \hat{\mathbf{t}}\left(s^{\prime}\right)\left\{\left(1+\frac{h^{2}}{R^{2}}\right)^{1 / 2}-1\right\} \approx
$$

where for $\Lambda / h \gg 1$ 


$$
J(x) \simeq \frac{1}{4}+\frac{1}{2} \ln (2 x)
$$

It follows that the curve-shortening equation in the presence of dipolar forces within the localized induction approximation is

$\frac{1}{\eta} \frac{\partial L}{\partial t} \simeq \oint_{\mathrm{d} s \kappa(s)}\left(\frac{2 \pi}{L}-\kappa(s)\right)\left[\gamma-\mu^{2} \ln \left(\frac{2 \Lambda(s) e^{1 / 2}}{h}\right)\right]$

Viewed this way, the velocity of curve-shortening may be thought of as a sum of contributions from segments of the interface. Since the natural scale of the cutoff $\Lambda(s)$ would be $1 /|\kappa(s)|$, those regions with high curvature and hence small $\Lambda$ will produce a higher effective line tension in eq 4.15 and vice versa. The early stages of shape evolution are then associated with higher effective tension.

The close correspondence between the Biot-Savart integral and the local curvature can be seen quite clearly in Figure 10 , in which we have plotted as a function of arc length both the local curvature and the Biot-Savart force $U_{\mu}$, obtained from eq $A 7$ by direct numerical computation, for the branched shape shown in the inset. A similar comparison may be made with the two contributions to the Lagrange multiplier, which flow to the fixed points given in eq 4.7.

\section{Fingering Instabilities and Domain Fission}

In the previous sections we have considered fluctuations about and relaxation toward a circular domain. Here we discuss an intriguing connection between the branching instabilities and the energetics of domain fission, a connection made apparent by the considerable calculational simplifications found in the ultrathin limit. We suggest viewing the branching process as a topology transition frustrated by barriers to fission. To do this, we compare at fixed Bond number the energies of a single circular domain of radius $R_{1}$ and of a set of smaller domains whose total volume is that of the first. The interactions between these smaller domains are neglected, as if they were infinitely far apart.

We obtain two main results from this analysis. First, the critical Bond number at which fissioning becomes energetically favored has the same logarithmic dependence on the domain radius as was found in the analysis for fingering instabilities, eq 2.11. This suggests that the essential physics of the instabilities may be understood in terms of the form of the selfenergy of dipolar domains. Our second result generalizes to arbitrary $n$ the earlier work of Keller, Korb, and McConnell ${ }^{8}$ on fissioning and the $n=2$ (elliptical) instability, who concluded that as a function of the Bond number, the energy of the noninteracting fissioned pair drops below that of the original circle before the circle becomes linearly unstable to elliptical distortions. In generalizing that analysis to arbitrary $n$, we consider two distinct fissioning processes: (i) fission into $n$ equivalent domains and (ii) fission into $n$ equivalent domains tilting the perimeter of the original circle plus one remaining central domain.

Equivalent Fission. If $A_{1}=\pi R_{1}^{2}$ is the area of the single domain, then the radius $R_{n}$ of the smaller circles must satisfy $A_{1}=n \pi R_{n}{ }^{2}$, so

$$
R_{n}=R_{1} / n^{1 / 2}
$$

In the ultrathin limit, the leading term in the dipolar energy of a domain of radius $R$, beyond that strictly proportional to the

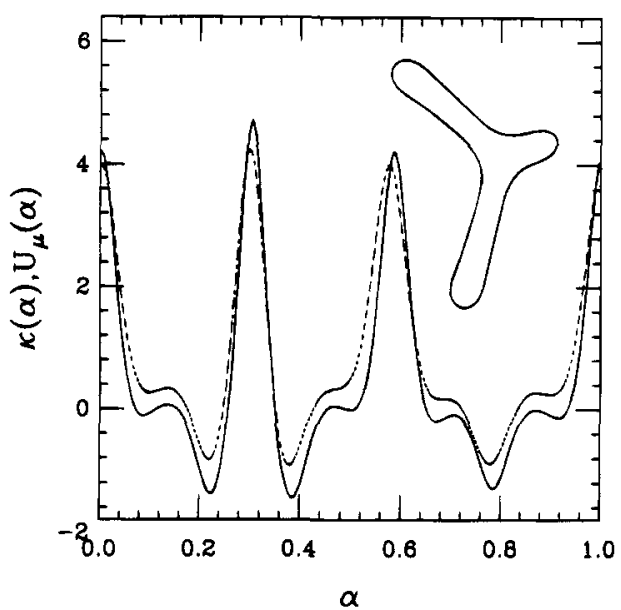

Figure 10. Two contributions to the normal velocity of the interface for a branched shape (inset). The curvature (solid line) is compared to the dipolar contribution (dashed line) given by eq $A 7$.

volume, is given by eq 2.5 ,

$$
\mathcal{E}_{\mu}(R) \simeq-2 \pi R \mu^{2} \ln \left(\frac{8 R}{h e^{1 / 2}}\right)
$$

while the line tension contribution is clearly $\mathscr{E}_{\gamma}(R)=2 \pi R \gamma$. It follows that the difference in energy $\Delta \mathcal{B}^{\prime}(n)$ between the $n$ noninteracting domains and the single domain is

$$
\begin{array}{r}
\Delta \mathcal{S}^{\prime}(n)=2 \pi R_{1}\left\{\left[\gamma-\mu^{2} \ln \left(\frac{8 R_{1}}{h e^{1 / 2}}\right)\right]\left(n^{1 / 2}-1\right)+\right. \\
\left.\mu^{2} n^{1 / 2} \ln \left(n^{1 / 2}\right)\right]
\end{array}
$$

Just as the linear stability analysis yielded the critical Bond number $N_{\mathrm{B}} *(n)$ at which the $n$-fold mode becomes energetically favorable relative to the unperturbed circle, so too in this calculation we obtain an estimate $N_{\mathrm{B}}{ }^{\prime}(n)$ for the energy crossing $\Delta \mathcal{O}^{\prime}(n)=0$,

$$
N_{\mathrm{B}}^{\prime}(n)=\frac{2}{\ln \left(8 R_{1} / h\right)-g^{\prime}(n)}
$$

with

$$
g^{\prime}(n)=\frac{1}{2}\left[1+\frac{n^{1 / 2}}{n^{1 / 2}-1} \ln (n)\right]
$$

The overall structure of eqs 5.4 and 5.5 is in close correspondence with the result (eqs 2.11 and 2.12) from the exact linear stability analysis. Figure 2 shows that $g^{\prime}(n)$ is quite close to $g(n)$ for small $n$ (particularly so for $n=2$, as observed by Keller et $a l^{8}$ ), but the deviations grow large for large $n$. Asymptotically for $n \gg 1$, we see that $g(n) \sim \ln (n)$, whereas $g^{\prime}(n) \sim \ln \left(n^{1 / 2}\right)$. We can trace the erroneous $n^{1 / 2}$ dependence to the assumption of equivalent fission, which views instability as a bulk phenomenon rather than a surface phenomenon.

Inequivalent Fission. In this case we determine the radii of the smaller domains by the tiling condition: $n 2 R_{n}=2 \pi R_{1}$. The area $A^{\prime}=\pi R^{\prime 2}$ of the one inequivalent domain is determined from the requirement of area conservation, $A=n A_{n}+A^{\prime}$. This yields

$$
R_{n}=\pi R_{1} / n \text { and } R^{\prime}=R_{1}\left(1-\pi^{2} / n\right)^{1 / 2}
$$

Clearly, this type of fission is logically consistent only if $A^{\prime}>$ 


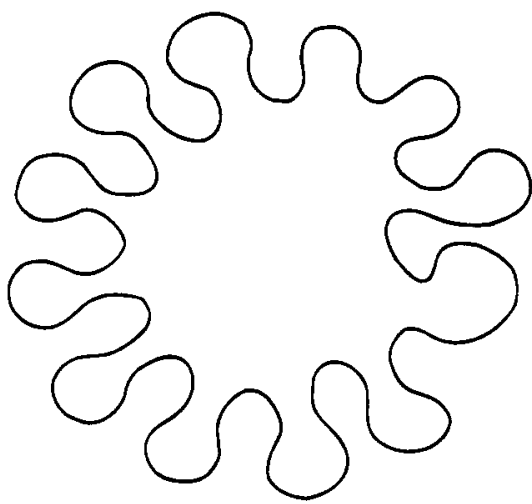

Figure 11. Early stage of fingering instability seen in experiments on a $2.5 \mathrm{~cm}$ ferrofluid domain in an applied magnetic field of $110 \mathrm{G}$ (ref 27). Note the close approach to inequivalent fissioning.

0 , or $n>10$ (so $\pi^{2} / n<1$ ). Repeating the calculation in eq 5.3 , we find the energy difference

$$
\begin{aligned}
& \Delta \mathcal{G}^{\prime \prime}(n)=2 \pi R_{1}\left\{[ \gamma - \mu ^ { 2 } \operatorname { l n } ( \frac { 8 R _ { 1 } } { h e ^ { 1 / 2 } } ) ] \left[\left(1-\pi^{2} / n\right)^{1 / 2}+\right.\right. \\
& \left.\pi-1]-\mu^{2}\left[\left(1-\pi^{2} / n\right)^{1 / 2} \ln \left(1-\pi^{2} / n\right)^{1 / 2}+\pi \ln (\pi / n)\right]\right\}
\end{aligned}
$$

A second estimate of the critical Bond number $N_{\mathrm{B}}{ }^{\prime \prime}(n)$, again from the energy crossing condition, yields

$$
N_{\mathrm{B}}^{\prime \prime}(n)=\frac{2}{\ln \left(8 R_{1} / h\right)-g^{\prime \prime}(n)}
$$

with

$g^{\prime \prime}(n)=\frac{\pi \ln (n / \pi)-\left(1-\pi^{2} / n\right)^{1 / 2} \ln \left(1-\pi^{2} / n\right)^{1 / 2}}{\left(1-\pi^{2} / n\right)^{1 / 2}+\pi-1}$

The function $g^{\prime \prime}(n)$ indeed has the same asymptotic behavior $g^{\prime \prime}(n) \sim \ln (n)$ as the exact $g(n)$, differing only by an additive constant, as shown in the linear-logarithmic plot of Figure 2. In comparing the exact result with the two approximations above, we see that, for each $n, g(n)>g^{\prime \prime}(n)>g^{\prime}(n)$, implying that the exact $N_{\mathrm{B}}{ }^{*}(n)$ is larger than those estimated from either fission analysis. When viewed as a function of increasing Bond number, the noninteracting fissioned state thus lies lower in energy than the circle before the circle becomes linearly unstable to fingering. Since this energetic comparison neglected the repulsive interactions between the fissioned domains, this suggests that there is a barrier to domain fission. Viewing the fission process as a caricature of fingering, it is plausible to conclude that the fingering instability is also first order. This conclusion is consistent with that of de Koker and McConnell, ${ }^{24}$ who found a barrier to domain fission for the $n=2$ mode by studying a one-parameter family of shapes which interpolated between a circle and a figure-eight.

Experiments on the shape transformations of magnetic fluid domains in Hele-Shaw flow reveal that the picture of finger instabilities as related to fissioning events is reasonable. Figure 11 shows a snapshot from the early stages of this instability in a recent experimental study, ${ }^{27}$ a fraction of a second after the application of a field well beyond the critical value. We see the domain boundary forming a well-defined set of circular extensions connected to a central region by narrow necks. Upon subsequent evolution those necks elongate rather than break, suggesting that in this purely deterministic flow the barriers to fission can not be overcome.

\section{Conclusions}

This paper has been concerned with the study of simple models for the thermal fluctuations and relaxation dynamics of amphiphilic monolayers. The experimental data on smallamplitude fluctuations about circular shapes are fully consistent with the predictions of a model in which the dipole density is presumed uniform and competes with a bare line tension. Dynamical studies of the relaxation toward circular shapes are qualitatively consistent with the simplest local model of interface dissipation in the presence of these competing forces. Of course, the observed behavior may equally well be due to a hydrodynamic effect not captured within that model, and recent work in that direction may shed light on this issue. ${ }^{31}$

Clearly, it is desirable that there be a more complete study of shape fluctuations and relaxation in the neighborhood of the branching instability to provide a more stringent test of the theoretical results. In this regard, studies on widely different domain sizes would be particularly useful. The nature of these fluctuations in stripe-like domains is also of interest in light of observations of "buckling instabilities" in amphiphilic monolayers. ${ }^{32}$

On a more general level, domains in amphiphilic monolayers may also serve as a useful model system for understanding thermally excited hopping of extended objects between local energetic minima. Such large-amplitude fluctuations have been seen in Langmuir monolayers ${ }^{33}$ and present an intriguing generalization of the "Kramers" problem (escape of a particle from a potential well) to a system with many degrees of freedom. As discussed in section $\mathrm{V}$, these shape transitions appear to be of first order, and the relevant barriers may indeed be comparable to thermal energy.

Acknowledgment. We are indebted to Michael Seul for extensive discussions concerning his experimental data and for important insights into pattern formation in Langmuir monolayers. M. Seul and S. A. Langer provided detailed comments and suggestions on the manuscript. We are grateful to A. O. Cebers, S. Erramilli, T. Gardner, and M. J. Shelley for ongoing collaborations, and to K. Y. Lee, H. M. McConnell, H. Stone, T. K. Vanderlick, and R. Mayer for communication of results prior to publication. The work of R.E.G. was supported in part by the Alfred P. Sloan Foundation and by a National Science Foundation Presidential Faculty Fellowship, Grant No. DMR9350227.

\section{Appendix A: Spectrum of Thermal Fluctuations}

Here we collect details of the calculation of the quantities $\Omega_{n}$ in eqs 2.8 and 2.9. Following Deutch and Low, ${ }^{13}$ we introduce a dimensionless small parameter $\epsilon$ to order the perturbation series,

$$
\xi(\varphi)=\epsilon \zeta^{(1)}(\varphi)+\epsilon^{2} \zeta^{(2)}(\varphi)+\ldots
$$

and similarly for the associated Fourier components $\zeta_{n}^{(1)}$ and $\xi_{n}^{(2)}$. 
First, expand the area up to quadratic order

$$
\begin{aligned}
& A= \frac{1}{2} \int \mathrm{d} \varphi \mathbf{r} \times \mathbf{r}_{\varphi} \\
& \simeq \pi R^{2}+\epsilon R \int \mathrm{d} \varphi \zeta^{(1)}+\epsilon^{2} R \int \mathrm{d} \varphi \zeta^{(2)}+ \\
& \frac{\epsilon^{2}}{2} \int \mathrm{d} \varphi \zeta^{(1)^{2}}+Q\left(\epsilon^{3}\right)
\end{aligned}
$$

Area conservation to order $\epsilon$ clearly requires that $\xi^{(1)}$ have zero mean, so $\zeta_{0}^{(1)}=0$, while at second order we obtain the constraint

$$
\int \mathrm{d} \varphi \xi^{(2)}=-\frac{1}{2 R} \int \mathrm{d} \varphi \xi^{(1)^{2}}
$$

Next, we observe that in the expansion of the line energy the perimeter has the form

$$
\begin{aligned}
L & =\int \mathrm{d} \varphi\left(\mathbf{r}_{\varphi} \cdot \mathbf{r}_{\varphi}\right)^{1 / 2} \\
& \simeq 2 \pi R+\epsilon \int \mathrm{d} \varphi \zeta^{(1)}+\epsilon^{2} \int \mathrm{d} \varphi \zeta^{(2)}+\frac{\epsilon^{2}}{2 R} \int \mathrm{d} \varphi \zeta_{\varphi}^{(1)^{2}}+Q\left(\xi^{3}\right)
\end{aligned}
$$

Substituting eq $A 3$ into eq $A 4$, and setting $\epsilon \equiv 1$, the line tension contribution to the excess energy is

$$
\begin{aligned}
\gamma\left(\left.L\right|_{A}-2 \pi R\right) & \simeq \frac{\gamma}{2 R} \int \mathrm{d} \varphi\left(\zeta_{\varphi}^{(1)^{2}}-\zeta^{(1)^{2}}\right) \\
& \simeq \frac{\pi \gamma}{2 R} \sum_{n=1}^{\infty}\left(n^{2}-1\right) \zeta_{n}{ }^{2}
\end{aligned}
$$

To determine the expansion of the dipole energy, we consider first arbitrary values of the cutoff $h$ and observe the simplification obtained by expanding the functional derivative of the energy about a circular shape. That derivative was computed in earlier work. ${ }^{14,19}$ If we define

$$
U_{\mu}=-\hat{\mathbf{n}} \cdot \frac{\delta \mathcal{O}_{\mu}}{\delta \mathbf{r}}
$$

then

$U_{\mu}(s)=\frac{2 \mu^{2}}{h^{2}} \oint_{\mathrm{d} s^{\prime}} \frac{\left(\mathbf{r}\left(s^{\prime}\right)-\mathbf{r}(s)\right)}{\left|\mathbf{r}\left(s^{\prime}\right)-\mathbf{r}(s)\right|} \times \hat{\mathbf{t}}\left(s^{\prime}\right)\left\{\left(1+\frac{h^{2}}{R^{2}}\right)^{1 / 2}-1\right\}$

Linearizing eq A7, using earlier results, ${ }^{14}$ and transforming to Fourier space, we obtain the coefficients $\Omega_{n}$ in eq 2.7 .

$$
\Omega_{n}=\frac{\pi}{R}\left\{\gamma\left(n^{2}-1\right)+\mu^{2} \mathscr{D}_{n}(p)\right\}
$$

where

$$
\begin{array}{r}
\mathscr{D}_{n}(p)=p^{2}\left\{\sum_{j=1}^{n} \frac{1}{2 j-1}-1+\frac{1}{k}\left[E+\left(k^{2}-1\right) K\right]-\right. \\
\left.p \int_{0}^{\pi / 2} \mathrm{~d} w \frac{\sin ^{2}(n w)}{\Delta(w)}\right\}
\end{array}
$$

$\Delta(w)=\left[1+p^{2} \sin ^{2}(w)\right]^{1 / 2}$, and $k$ is defined below eq 2.4 .
Analytical progress in the limit $p \gg 1$ is facilitated by Thiele's results ${ }^{34}$ on integrals of the type appearing in eq A9, yielding eq 2.9 .

\section{Appendix B: Details of the Numerical Method}

Cutoff Contributions. In this section we describe the numerical method used to study shape relaxation, paying particular attention to the treatment of the cutoff $h$ which enters the energy and Biot-Savart force integrals 2.2 and A7. ${ }^{35}$ Any discretization of the boundary used in the equation of motion (eq 4.1) has a resolution limited by the grid spacing $\Delta s$. If we wish to make contact with the exact analytical results for the energy and dipolar pressure for simple geometric shapes, it is desirable to include in the computation the contributions arising from integrations up to the grid size. In specializing to the ultrathin limit, we shall thus assume that not only is $p \gg 1$ but also that the curvature $\kappa$ of any shape of interest satisfies

$$
h \kappa \ll 1
$$

It is then possible to introduce a cutoff $\Lambda$, for instance the grid spacing, at an intermediate scale satisfying $h \ll \Lambda \ll 1 / \kappa$. Then the scalar product in eq 2.2 ,

$$
\hat{\mathbf{t}}(s) \cdot \hat{\mathbf{t}}\left(s^{\prime}\right) \simeq 1-\frac{1}{2}\left(s-s^{\prime}\right)^{2} \kappa^{2}+\ldots
$$

is well-approximated by unity for $\left|s-s^{\prime}\right| \leq \Lambda$. For larger separations, $\Phi$ conforms to its asymptotic behavior $\Phi(\xi) \simeq 1 / 2 \xi$ and we may write

$$
\begin{aligned}
& \epsilon_{\mu} \simeq-\frac{1}{2} \mu^{2} \oint_{\mathrm{d} s} \oint_{\mathrm{d} s^{\prime}} \frac{\hat{t}(s) \cdot \hat{\imath}\left(s^{\prime}\right)}{\left|\mathbf{r}(s)-\mathbf{r}\left(s^{\prime}\right)\right|}-\frac{1}{2} \mu^{2} L I(\Lambda / h) \\
& \left|s-s^{\prime}\right| \geqslant \Lambda
\end{aligned}
$$

The double integral has the standard form of the self-induction of a current-carrying wire, and the cutoff contribution is

$$
\begin{aligned}
I(\Lambda / h) & =\int_{0}^{\Lambda} \mathrm{d} s \Phi(s / h) \\
& =\frac{1}{2} x^{2}-\frac{1}{2} x\left(1+x^{2}\right)^{1 / 2}+x \sinh ^{-1}(1 / x)+\frac{1}{2} \sinh ^{-1}(x)
\end{aligned}
$$

The cutoff procedure described in section IV (eqs 4.11 and 4.12) may be used on the Biot-Savart integral to obtain the decomposition

$$
U_{\mu}=-\mu^{2} \oint_{\left|s-s^{\prime}\right| \geqslant \Lambda} \frac{\left(\mathbf{r}(s)-\mathbf{r}\left(s^{\prime}\right)\right)}{\left|r(s)-\mathbf{r}\left(s^{\prime}\right)\right|^{3}} \hat{t}\left(s^{\prime}\right)+2 \mu^{2} \kappa(s) J(\Lambda / h)
$$

where, analogously to eq $\mathrm{B} 4$, the cutoff contribution is

$$
\begin{aligned}
J(x) & =\int_{0}^{x} \mathrm{~d} x^{\prime}\left\{\left(1+x^{2}\right)^{1 / 2}-x\right\} \\
& =\frac{1}{2} x\left(1+x^{2}\right)^{1 / 2}-\frac{1}{2} x^{2}+\frac{1}{2} \ln \left[x+\left(1+x^{2}\right)^{1 / 2}\right]
\end{aligned}
$$

In numerical computations we are particularly interested in the case $N h \gg 1$, where the approximations

$$
I(x) \simeq \frac{3}{4}+\frac{1}{2} \ln (2 x) \text { and } J(x) \simeq \frac{1}{4}+\frac{1}{2} \ln (2 x)
$$

hold. These approximations are highly accurate even for $N / h$ $\simeq 1$. 


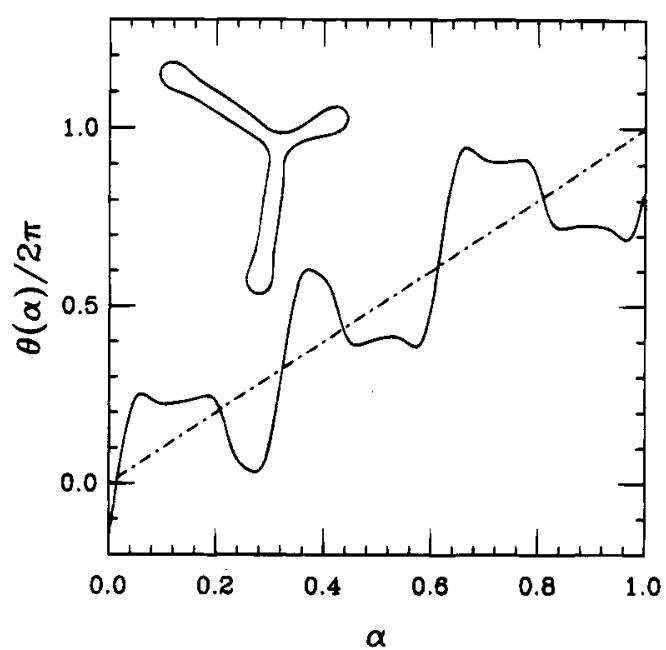

Figure 12. Tangent angle (solid line) associated with a branched domain, and uniform winding solution for a circle (dashed line).

Tangent Angle Dynamics. To study the boundary evolution we have employed some basic results from the differential geometry of curve motion discussed in earlier work, ${ }^{14}$ with some important modifications. A particularly convenient method to obtain the boundary evolution is to work with the tangent angle $\theta(s)$, related to the curvature by $\kappa(s)=\partial \theta / \partial s$. If the equation of motion for the boundary is in the form of eq 4.3, $\theta$ evolves as $^{30}$

$$
\frac{\partial \theta}{\partial t}=-\frac{\partial U}{\partial s}+\kappa W
$$

As described below, it is convenient to use a spectral method to solve the resulting partial differential equation for $\theta$. However, the function $\theta$ itself is not appropriate for this method, for it acquires a net angle $2 \pi$ for each traversal of the boundary and is therefore not periodic in $s$. Figure 12 shows the function $\theta$ for the branched initial condition of Figure $7 \mathrm{a}$. The dashed line represents the "winding" $\theta=2 \pi s / L$ of $\theta$ that describes a closed circular domain. It is natural then to define the $2 \pi$-periodic function $\psi$ with zero mean,

$$
\theta(\alpha, t)=2 \pi \alpha+\psi(\alpha, t)
$$

adopting the relative arc length gauge $\alpha=s / L$. From eq B8 we see that $\psi$ evolves as

$$
\frac{\partial \psi}{\partial t}=-\frac{1}{L} \frac{\partial U}{\partial \alpha}+\left(\frac{2 \pi}{L}+\frac{1}{L} \frac{\partial \psi}{\partial \alpha}\right) W
$$

The choice of $W$ consistent with the relative arc length gauge has been given previously, ${ }^{14,30}$

$$
W(\alpha)=L\left(\alpha \int_{0}^{1} \mathrm{~d} \alpha^{\prime} \kappa U-\int_{0}^{\alpha} \mathrm{d} \alpha^{\prime} \kappa U\right)
$$

Pseudospectral Method. Next we observe that the contribution to eq B10 arising from the curvature of the interface contains the highest derivative with respect to $\alpha$ in the $\psi$ evolution equation, and appears linearly in the form

$$
\frac{\partial \psi}{\partial t}=\frac{\gamma}{L^{2}} \frac{\partial^{2} \psi}{\partial \alpha^{2}}+\text { nonlinear terms }+ \text { nonlocal terms }
$$

This property allows us to employ an algorithm, motivated by recent work on vortex methods in hydrodynamics, ${ }^{36}$ that overcomes many of the "stiffness" problems associated with diffusive PDEs and allows for efficient integration of the equation of motion. Consider first the general partial differential equation for a scalar quantity $q(x, t)$, a function of a single spatial dimension and time:

$$
\frac{\partial q}{\partial t}=\mathcal{L}\left(\frac{\partial}{\partial x}\right) q+N[q]
$$

where $\mathscr{L}$ is a linear operator and $N$ is nonlinear. Were it the case that $N=0$, then the dynamics are exactly solvable in Fourier space. With $\hat{q}(k, t)$ being the spatial Fourier transform of $q(x, t)$, the equation of motion in momentum space for $N \equiv$ 0 is

$$
\frac{\partial \hat{q}(k, t)}{\partial t}=w(k) \hat{q}(k, t), \quad w(k)=\mathscr{P}(i k)
$$

Then, for the purely linear case, if we know $\hat{q}(k, t)$, we obtain its value at a later time $t+\Delta t$ exactly as

$$
\hat{q}(k, t+\Delta t)=\mathrm{e}^{w(k) \Delta t} \hat{q}(k, t)
$$

The exponentiation of the growth rate $w(k)$ in eq B 15 plays a useful role in guaranteeing stability for a diffusive linear operator $\left(w(k)=-\gamma k^{2}\right)$. The usual stability considerations ${ }^{37}$ would require a time step $\Delta t$ such that for large values of momentum (near the Brillouin zone edge $k_{\max }=\pi / a$, with $a$ being the lattice spacing in real space) the quantity $k^{2} \Delta t$ be less than unity. This requires an extremely small time step, rendering the calculation prohibitively slow. Here, even if $k_{\max }^{2} \Delta t>1$, the calculation is stable due to the incorporation of the exact dynamics of the linear operator, namely, the exponential damping at high momentum.

To incorporate the exact information of eq B15 into dynamics with a nonlinearity, we assume that the highest order spatial derivative appears in the linear operator (as is the case in the $\psi$ dynamics) and treat the nonlinear terms pseudospectrally, defining $\hat{N}(k, t)=\mathscr{F}[N(q(x, t))]$, with $q(x, t)$ being obtained from $\hat{q}(k, t)$ by inverse fast Fourier transformation. The equation of motion (eq B13) then becomes

$$
\frac{\partial \hat{q}(k, t)}{\partial t}-w(k) \hat{q}(k, t)=\hat{N}(k, t)
$$

Now define

$$
\hat{Q}(k, t)=\mathrm{e}^{-w(k) t} \hat{q}(k, t)
$$

and multiply eq $\mathrm{B} 16$ by the exponential factor, yielding

$$
\frac{\partial \hat{Q}_{t}(k, t)}{\partial t}=\mathrm{e}^{-w(k) t} \hat{N}\left[\hat{Q} \mathrm{e}^{w(k) t}\right]
$$

This equation may be solved by any of a number of methods. In the simplest Euler method, we have

$$
\frac{\hat{Q}(k, t+\Delta t)-\hat{Q}(k, t)}{\Delta t} \simeq \mathrm{e}^{-w(k) t} \hat{N}\left(\hat{Q}(k, t) \mathrm{e}^{w(k) t}\right)
$$

which yields

$$
\hat{q}(k, t+\Delta t)=\mathrm{e}^{w(k) \Delta t}[\hat{q}(k, t)+\Delta t \hat{N}(k, t)]
$$

The exponential factor thus provides damping of the highfrequency modes, lending stability to the method.

The time evolution of the function $\psi$ corresponding to the boundary evolution in Figure $7 \mathrm{a}$ is shown in Figure 13. We see in the early stages the relaxation of short-wavelength modes, leaving only the $n=2$ mode to decay in the late stages, 


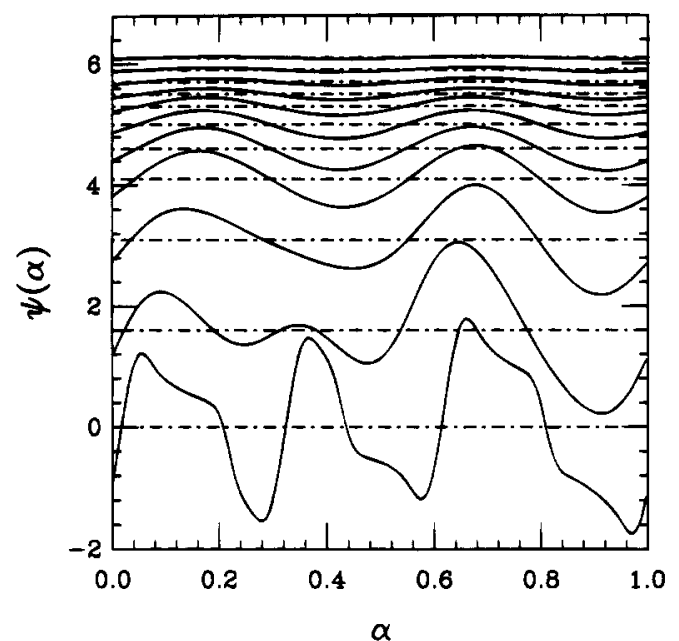

Figure 13. Evolution of the tangent angle function $\psi$ corresponding to the shape evolution in Figure 7.

corresponding to the relaxation of an elliptical shape to a circle. The considerable simplification in the shape evolution obtained by employing the $\psi$ representation is readily apparent in comparing the simple mode relaxation in Figure 13 with the rather complex shape evolution to which it corresponds.

\section{References and Notes}

(1) McConnell, H. M.; Rice, P. A.; Benvegnu, D. J. J. Phys. Chem. $1990,94,8965$.

(2) Benvegnu, D. J.; McConnell, H. M. J. Phys. Chem. 1992, 96, 6820.

(3) Seul, M.; Sammon, M. J. Phys. Rev. Lett. 1990, 64, 1903.

(4) Seul, M. Physica A 1990, 168, 198.

(5) Seul, M. J. Phys. Chem. 1993, 97, 2941.

(6) Seul, M.; Sammon, M. J.; Monar, L. R. Rev. Sci. Instrum. 1991 62,784 . 3673

(7) Andelman, D.; Brochard, F.; Joanny, J. J. Chem. Phys. 1987, 86,

(8) Keller, D. J.; Korb, J. P.; McConnell, H. M. J. Phys. Chem. 1987. $91,6417$.

(9) McConnell, H. M.; Moy, V. T. J. Phys. Chem. 1988, 92, 4520.

(10) McConnell, H. M. J. Phys. Chem. 1990, 94, 4728.

(11) Vanderlick, T. K.; Möhwald, H. J. Phys. Chem. 1990, 94, 886.

(12) Cebers, A. O. Magnetohydrodynamics 1989, 25, 149

(13) Deutch, J. M.; Low, F. E. J. Phys. Chem. 1992, 96, 7097.

(14) Langer, S. A.; Goldstein, R. E.; Jackson, D. P. Phys. Rev. A 1992 46,4894

(15) Rosenweig, R. E. Ferrohydrodynamics; Cambridge University Press: Cambridge, 1985 .

(16) Tsebers, A. O.; Maiorov, M. M. Magnetohydrodynamics 1980, 16 21.

(17) Boudouvis, A. G.; Puchalla, J. L.; Scriven, L. E. J. Colloid Interface Sci. 1988, 124, 688.

(18) Dickstein, A. J.; Erramilli, S.; Goldstein, R. E.; Jackson, D. P.; Langer, S. A. Science 1993, 261, 1012.

(19) Jackson, D. P.; Goldstein. R. E.; Cebers, A. O. Phys. Rev. E 1994. $50,298$.

(20) McConnell, H. M. J. Phys. Chem. 1992, 96, 3167.

(21) Batchelor, G. K. An Introduction to Fluid Dynamics; Cambridge University Press: Cambridge, 1967. The original notion of the localized induction approximation is due to Da Rios: Da Rios, L. S. Rend. Circ. Mat. Palermo 1906, 22, 117, and rediscovered several times since. For a brief history of the LIA, see: Ricca, R. L. Nature 1991, 352, 561 and references therein. We are indebted to $R$. L. Ricca for bringing these works to our attention.
(22) The application of the localized induction approximation to the motion of curves in the plane follows closely a study of the motion of vortex patches; see: Goldstein, R. E.; Petrich, D. M. Phys. Rev. Lett. 1992, 69,555 .

(23) Lee, K. Y. C.; McConnell, H. M. J. Phys. Chem. 1993, 97, 9532.

(24) de Koker, R.; McConnel1, H. M. J. Phys. Chem. 1993, 97, 13419.

(25) Lorenz, L. Wied. Ann. 1879, 7, 161

(26) The exact results for the critical Bond numbers for the $n=2$ and $n=3$ modes are

$$
\begin{array}{r}
N_{\mathrm{B}}(2)=\frac{9 k\left(1-k^{2}\right)}{k^{3}+\left(8-11 k^{2}+3 k^{4}\right) K+\left(7 k^{2}-8\right) E} \\
N_{\mathrm{B}}(3)=\frac{15 k^{3}\left(1-k^{2}\right)}{k^{5}+8\left(2-3 k^{2}+k^{4}\right) K-\left(k^{4}-16 k^{2}+16\right) E}
\end{array}
$$

where, as in eq $2.4, k^{2}=p^{2} /\left(1+p^{2}\right)$ and $E(k)$ and $K(k)$ are complete elliptic integrals.

(27) Gardner, T.; Erramilli, S.; Goldstein, R. E. Interfacial Pattern Formation and the Biot-Savart Law, 1994, preprint.

(28) Reference 10 derives the relation for the radius for the $n$ th-order instability:

$$
R^{(n)}=\frac{\delta}{4} \mathrm{e}^{\gamma / \mu^{2}} \mathrm{e}^{Z_{n}}
$$

where $\delta$ is the sharp cutoff and a recursion relation for the quantites $Z_{n}$ is given. This result is fully consistent with eq 2.13 with the identifications $Z_{n}=3 / 2+g(n), \delta=h e^{3 / 2} / 2$.

(29) Cebers, A. O. Private communication, 1993.

(30) Brower, R. C.; Kessler, D. A.; Koplik, J.; Levine, H. Phys. Rev. A 1984, 29, 1335

(31) Stone, H. A.; McConnell, H. M. Proc. R. Soc. London, A, in press.

(32) Stine, K. J.; Knobler, C. M.; Desai, R. C. Phys. Rev. Lett. 1990. 65,1004 .

(33) Seul, M. Private communication, 1993.

(34) Thiele, A. A. Bell Syst. Tech. J. 1969, 48, 3287. This paper contains a number of useful results on the asymptotic behavior of integrals of the type appearing in eq A9. Here we make use of the relation

$$
\begin{aligned}
& \int_{0}^{\pi / 2} \mathrm{~d} w \frac{\sin ^{2}(n w)}{\left[x+\sin ^{2}(w)\right]^{1 / 2}} \simeq \sum_{j=1}^{n} \frac{1}{2 j-1}+\frac{1}{4}\left|-n^{2} \ln \right| \frac{16}{x} \mid-2 n^{2}+ \\
&\left.\left(4 n^{2}-1\right) \sum_{j=1}^{n} \frac{1}{2 j-1}\right)+Q\left(x^{2}, x^{2} \ln (x)\right)
\end{aligned}
$$

(35) Mayer, M. A.; Vanderlick, T. K. Langmuir 1992, 8, 3131, describes alternative computational schemes for the evaluation of the dipolar energies. See also: Mayer, M. A.; Vanderlick, T. K. Calculation of Shapes of Dipolar Domains in Insoluble Monolayers: Analysis of Shape Stability and Transitions, 1993, preprint.

(36) Hou, T. Y.; Lowengrub, J.; Shelley, M. J. J. Comput. Phys., in press.

(37) Press, W. H.; Teukolsky, S. A.; Vetterling, W. T.; Flannery, B. P. Numerical Recipes in C, 2nd ed.; Cambridge University Press: Cambridge, 1992 